\title{
Electron diffraction studies of supersonic jets. VI. Microdrops of benzene
}

\author{
Edward J. Valente and Lawrence S. Bartell \\ Department of Chemistry, University of Michigan, Ann Arbor, Michigan 48109
}

(Received 28 September 1983; accepted 4 November 1983)

\begin{abstract}
Large clusters of benzene were generated in supersonic flow through a Laval nozzle with carrier gases $\mathrm{He}, \mathrm{Ne}$, or Ar. Benzene concentration and carrier pressures were varied over wide ranges. Diffraction patterns recorded from $s=0.8$ to $12 \AA^{-1}$ revealed strong intermolecular interference features indicative of supercooled bulk liquid at $100-200 \mathrm{~K}$. Computer simulations of nucleation and cluster growth were carried out and agreed with the following observations. An initial benzene partial pressure of $\sim 0.1$ bar expanded through a nozzle of our design requires a carrier pressure of 2-3 bar to give reasonably complete condensation. Cluster diameter and fraction condensed decrease if (1) benzene mole fraction is reduced at constant carrier pressure, (2) carrier pressure is reduced at constant benzene partial pressure, and (3) carrier molecular weight is decreased. The simulations identify the factors accounting for the trends. Cluster intensities are compared with those calculated for small clusters proposed by Williams, small crystal fragments, and bulk fluid according to the statistical mechanical algorithm RISM. None of the models reproduce observed results within experimental error but comparisons provide information about size, temperature, and structure. Intrinsic deficiencies of RISM are indicated by the present experiments.
\end{abstract}

\section{INTRODUCTION}

In a previous paper in this series we described the formation of benzene clusters in supersonic expansions with monatomic carrier gases. ${ }^{1}$ Electron diffraction patterns of the cold clusters in the range $s=2.5-13 \AA^{-1}$ showed elements of similarity with $\mathrm{x}$-ray patterns of bulk liquid ${ }^{2}$ but implied smaller thermal motions due to lower temperature. This method for the production of liquid-like samples circumvents the problem of severely limited penetrability of matter by electrons encountered in conventional studies of condensed phases by electron diffraction and offers, as well, advantages over $\mathrm{x}$-ray and neutron diffraction in resolving power, counting statistics, and sensitivity. New auxiliary equipment enables us to record diffraction features well inside the range previously studied by electron diffraction, and makes accessible the prominent inner diffraction features characterizing long range order in liquids and crystals.

In addition to experiment, several theoretical approaches have been applied to the investigation of condensed benzene and yield information from which diffraction intensities can be computed. Williams has calculated the structures of a number of small benzene clusters ${ }^{3}$ by minimization of the intermolecular potential energies. Several liquid simulations have recently been carried out using the reference interaction site model (RISM) of Lowden and Chandler. ${ }^{4}$ This theory, which originally used hard-sphere potentials, has had some success in describing diffraction features of benzene $e^{2,4}$ and tetrahedral molecules, ${ }^{5}$ as well as simpler molecules. ${ }^{6} \mathrm{~A}$ modification of this approach introduces more realistic interaction energies. ${ }^{7}$

In our previous diffraction study of benzene clusters formed in supersonic expansions, we described the experimental method and made preliminary comments on the patterns obtained. Here we present a more extensive series of experiments on benzene mixed with helium, neon, or argon carriers at various compositions and pressures. The resulting cluster signals are compared with theoretical patterns computed for several models. We also relate trends observed in our experiments with those implied by classical nucleation theory. Lastly, we carry out RISM calculations with modified potential functions and contrast results with those obtained in our experiments.

\section{EXPERIMENTAL}

The apparatus and methods of analysis have been outlined previously. ${ }^{1,8}$ A completely new set of data has been collected. Benzene partial pressure was controlled by adjusting the temperature of a bath surrounding a brass mixing tank containing benzene (certified A.C.S. reagent grade, Fisher Scientific) which was in contact with a glass wool plug that served as a filter and a wick. Helium, neon, and argon ( $>99.99 \%$, Air Products Ltd.) were used without further purification. These inert carrier gases at various pressures were passed over the benzene and through the glass wool on the way to the diffraction unit. Gas mixtures so obtained were expanded at ambient temperature $(298 \mathrm{~K})$ through our glass nozzle no. 6, a conical Laval nozzle the shape of which has been given elsewhere. ${ }^{8}$ Expansion conditions are listed in Table I. Patterns of $40 \mathrm{keV}$ electrons scattered by clusters about $12 \mathrm{~mm}$ downstream of the nozzle exit were recorded on $4 \times 5$ in. Kodak medium projector slides. The beam current was about $60 \mathrm{nA}$ for all plates, except some for neat benzene. A liquid nitrogen cold trap $130 \mathrm{~mm}$ from the nozzle accumulated disks of condensed benzene of diameter $4 \mathrm{~mm}$ with helium, $15 \mathrm{~mm}$ with neon, and $22 \mathrm{~mm}$ with argon carriers, illustrating differential effects of mass fractionation. Intensities from plates taken under the same conditions were averaged and leveled as outlined previously. ${ }^{1}$ Intensities for 
TABLE I. Experimental conditions of nozzle expansions. Reciprocals of relative diffraction exposures are proportional to scattering power of the skimmed supersonic jet.

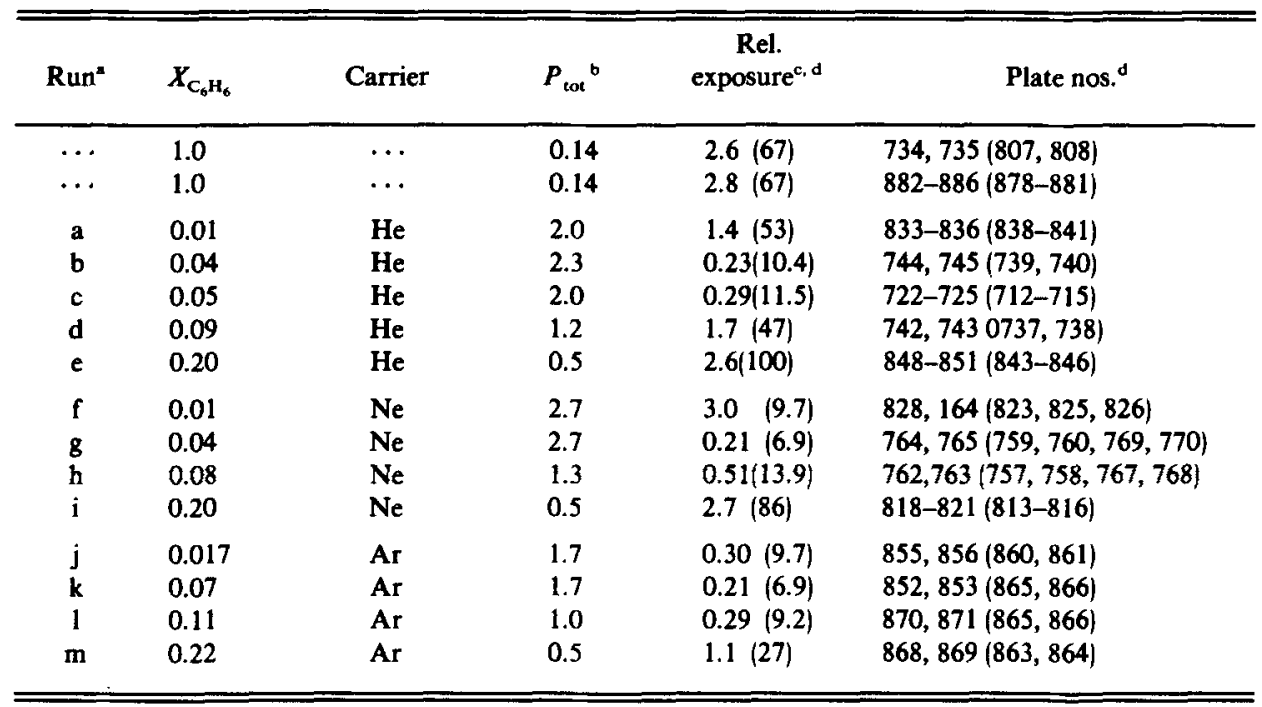

"Letter corresponds to label in Fig. 1.

${ }^{b}$ Total stagnation pressure, in bar.

'Time in $\mathbf{s}$ for $100 \mathrm{nA}$ electron beam to produce pattern of standard absorption at point of maximum density (normalized to unit absorbance).

d Parentheses, $r^{2}$ sector; without parentheses, $r$ sector.

representative plates are available as supplementary material. 9

Both $r^{1}\left(s=0.8-4.2 \AA^{-1}\right)$ and $r^{2}\left(s=2.4-12 \AA^{-1}\right)$ sectored data were collected for each set of expansion conditions. Microdensitometer readings of the plates were at intervals of $\Delta s=0.03 \AA^{-1}$. Intensities from each sectored range were blended into a single appropriately scaled composite intensity at intervals of $\Delta s=\pi / 20$.

All illustrations of cluster signals have been corrected by subtraction of a reproducible "systematic residual" function established in experiments with neat benzene. Such a function has been found to compensate effectively for deficiencies in the simplified electron distributions incorporated in the theoretical diffraction intensities. ${ }^{10}$

Benzene was assumed to be a regular hexagon with $\mathrm{C}-\mathrm{C}$ length $\left(r_{g}\right)$ of $1.399 \AA$ and $\mathrm{C}-\mathrm{H}$ length $1.101 \AA .{ }^{11}$ Because the effective camera height, approximately $214 \mathrm{~mm}$, cannot be measured directly with precision and may depend on the offaxis distribution of sample gas, a scale factor was allowed to refine and absorb this uncertainty. Other details of the molecular model and a damping term introduced to correct for the broadened cluster beam are documented in an earlier paper.'

\section{DIFFRACTION INTENSITIES}

Cluster signals are derived from leveled intensities by subtraction of molecular interference terms (calculated to correspond to the benzene monomer) and the carrier (mole fraction adjusted to compensate for mass fractionation). Intensities were also divided by a smooth background function and, finally, the systematic residual function was subtracted. A local cubic polynomial was fit to values of the cluster signal and interpolated to the desired $s$ values. Function $M(s)$ so derived, then, is the ratio of the benzene intermolecular scat- tering to the total atomic scattering by all benzene molecules whether condensed or not. When multiplied by variable $s$ it becomes the scattering signal whose Fourier transform is closely related to the interatomic pair correlation function. Signals from benzene clusters formed in helium, neon, and argon are illustrated in Fig. 1.

Particularly in the low angle region where electron data were unavailable previously, the cluster intensities pleasingly resemble those obtained in $\mathrm{x}$-ray studies on bulk liquid benzene. They differ in the prominence of the features at

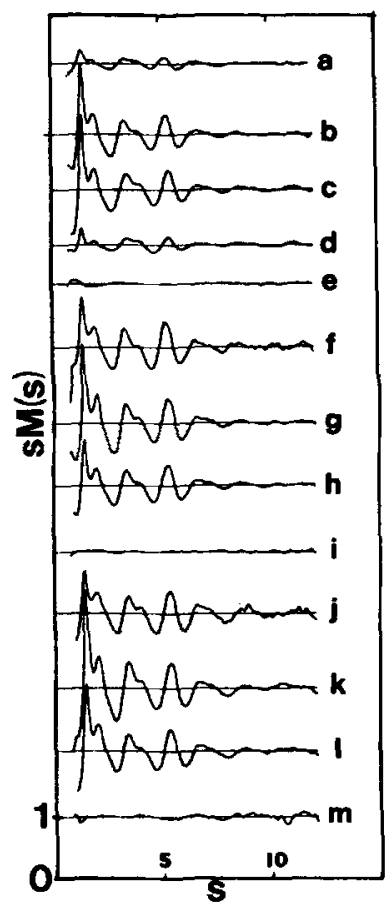

FIG. 1. Experimental benzene cluster structure functions (intermolecular interference patterns) obtained over a wide range of benzene mole fraction and carrier pressure. (a)-(c) Helium carrier. (f)(i) Neon carrier. (j)-(m) Argon carrier. Conditions and cluster characteristics identified in Tables I and II. 
higher angles where interference fringes are damped less rapidly for the colder scatterer. It is clear that even for the expansions seeded with the lowest subject mole fractions, electron diffraction is a sensitive measure of the cluster structure function despite the presence of considerable carrier scattering. For the expansions in helium one sees the growth of the cluster signal as the subject mole fraction is increased from 0.01 at constant total pressure. Higher subject mole factions ultimately show diminished cluster signals, but the corresponding total pressures are lower, limited by the vapor pressure of benzene at room temperature. Signals are clearly not crystalline patterns, for which DebyeScherrer rings would appear at low angle and well within the resolution of our diffraction plates (Fig. 7 of Ref. 8).

In the following sections, we discuss systematic trends in our results and examine several approaches of utility in piecing together the structure, size, and temperature of the benzene clusters.

\section{NUCLEATION CALCULATIONS}

Treatments of homogeneous nucleation in continuous nozzle flow have been applied in a number of studies of supersonically generated cluster beams. ${ }^{12-15}$ Potential benefits of modeling the formation of clusters are the inference of temperatures and sizes of clusters produced and the formulation of guidelines for effects of changing conditions and nozzle design. While comparatively simple diffraction measures of temperature and size are available for crystallites, corresponding measurements for amorphous materials are less straightforward. For this reason we have undertaken model calculations of nucleation and cluster growth even though the theory of homogenous nucleation is, at best, approximate. It is not our purpose here to describe in detail our computational procedures but only to compare experimental trends with those implied by model calculations. Therefore we only sketch our approach in outline. Our basis is the work of Oswatitsch ${ }^{16}$ as embodied in the computer program of $\mathrm{Wu}^{12}$ in Wu's "nonisothermal" variant. Although the governing relations follow those of $\mathrm{Wu}$, whose program is for large nozzles and neglects boundary layer effects, our algorithm is different in design. Our treatment of boundary layer effects, which become large in micronozzles, departs from that of Stein (who also introduces empirical strategems to render the problem tractable ${ }^{17}$ ) but yields similar results. Briefly, we assume that individual segments of length in the compressible flow ${ }^{18}$ are subject to pipe friction ${ }^{18}$ modeled after Stanton and Parnell. ${ }^{19}$ To take into account the fact that our nozzle is divergent and not a parallel walled pipe, we multiply the friction factor by a constant $(0.17$ in the tabulated runs) to make the effluent Mach numbers of the model calculations simulate those of experiment. ${ }^{17}$

For purposes of illustration we adopted a cluster density of $1.05 \mathrm{~g} / \mathrm{cm}^{3}$, vapor pressure $p_{\infty}$ and heat of vaporization extrapolated to $150 \mathrm{~K}$ from the liquid data of Yaws, ${ }^{20}$ then propagated $p_{\infty}(T)$ about $150 \mathrm{~K}$ via the Clausius-Clapeyron equation, and extrapolated $\sigma(T)$ for liquid using Quale's ${ }^{21}$ parameters. Surface tension was multiplied by Tol-

TABLE II. Calculated quantities related to cluster formation and observed heights of diffraction features (top $2 / 3$ of table). Corresponding quantities for model clusters and bulk liquid (bottom $1 / 3$ of table).

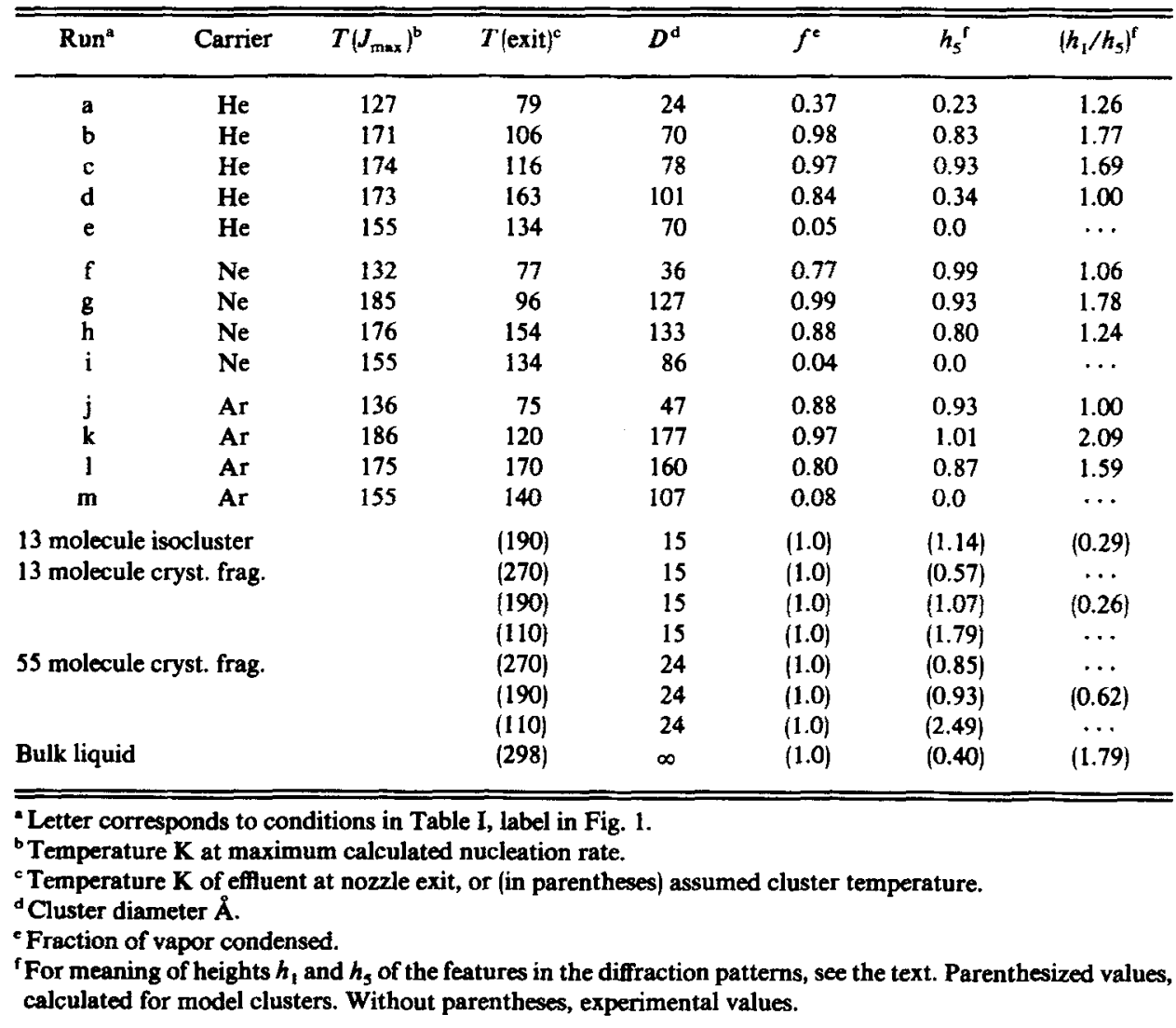


man's correction factor for droplet size. This factor was taken, very crudely, as $(1-2 / r)$ with drop radius $r$ in $\AA$ for $r>3.5 \AA$ (as it invariably was). Gas viscosities $\mu(T)$ were assumed to be those of the carrier gases, and Sutherland parameters for $\mu(T)$ appropriate for the range $50-250 \mathrm{~K}$ were determined from tabulated gas viscosities. ${ }^{22}$ Stein's nucleation rate correction factor ${ }^{17} \Gamma=10^{5}$ for micronozzle flow was adopted.

Selected results of the nozzle flow calculations are listed in Table II together with some experimental observations to be discussed later. Stagnation pressures, temperatures, and compositions for the tabulated runs correspond to those of the experiments presented in Fig. 1.

\section{SMALL CLUSTERS}

Although the sharp feature in the cluster scattering at $s=1.3 \AA^{-1}$ is indicative of substantial cluster diameter, it is instructive to compare observed intensities with those of proposed small cluster models. In a recent study of large water clusters the most successful model incorporated results of a molecular dynamics calculation including only 20 molecules. ${ }^{23}$ Williams ${ }^{3}$ has computed a number of benzene cluster configurations corresponding to intermolecular potential energy minima for clusters comprised of 2 to 15 molecules. Starting configurations were based on arrangements in orthorhombic crystal fragments. Using amplitudes of vibration listed in Ref. 1 we have calculated scattered intensities for the two Williams clusters with a closed first coordination shell, namely, the 13-molecular cluster closely related to the crystal configuration and the 13-molecule isocluster. These are plotted in Figs. 2(a) and 2(b) where temperatures are assumed to be about $190 \mathrm{~K}$. Calculated intensities for the 13 clusters are seen to be similar to those of the 55 molecule crystal fragment [Fig. 2(c)] at the same temperature. The experimental cluster intensity [Fig. 2(e)] and that measured for bulk liquid benzene by x-ray diffraction [Fig. 2(d)] are markedly different from those of the cluster models, most notably in the low angle region. Nevertheless, the approximate agreement in position and signs of the interference features suggests that the condensates have structures related in gross aspects but not in detail to those of the cluster and

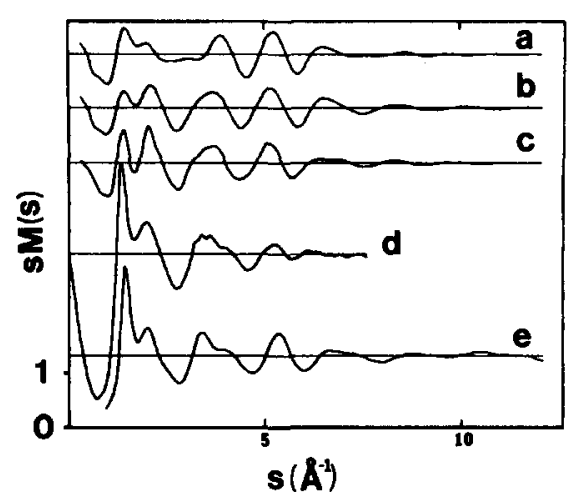

FIG. 2. Benzene intermolecular interference patterns. (a)-(c) Calculated, respectively, for Williams 13 molecule "cluster" and "isocluster" and for a 55 molecule crystal fragment (Ref. 1), all nominally at $190 \mathrm{~K}$. (d) Narten's Xray intensities (Ref. 2). (e) Electron diffraction intensities, present investigation. crystal models. Selected characteristics of the calculated intensities are listed in Table II.

\section{SIMULATIONS VIA REFERENCE INTERACTION SITE MODEL}

The reference interaction site model (RISM) of Lowden and Chandler $^{4}$ provides a plausible description of the structures of several simple molecular liquids, ${ }^{5,20,24}$ and has been applied to bulk liquid benzene by Narten. ${ }^{2}$ In Narten's model, where interatom potentials were represented by hardsphere interactions, a rough agreement between the RISM and the room temperature experimental structure functions was obtained. Narten attributed discrepancies to inadequacy of the RISM calculations. Pair correlation functions derived from the analysis, while approximate, bore similarities to those derived from the crystal structure $e^{6,25,26}$ and from molecular dynamics calculations ${ }^{27}$ on the liquid. A modification of the RISM routine incorporating more realistic potentials and temperature dependence was subsequently introduced by Johnson and Hazoume. ${ }^{28}$ Intermolecular potential functions suitable for preliminary RISM calculations are those derived by Williams ${ }^{29}$ to account for crystallographic data on aromatic molecules. Parameters for his Buckingham interaction potential energies are given in Table III.

Using the Williams potential parameters, we computed the RISM structure function for bulk benzene at $298 \mathrm{~K}$ where the number density is $0.006738 / \AA^{3}$. A regular hexagon with six $\mathrm{C}$-atom and six $\mathrm{H}$-atom sites at $\mathrm{C}-\mathrm{C}$ distance $1.397 \AA$ and $\mathrm{C}-\mathrm{H}$ distance $1.102 \AA$ comprised the molecular model. Hydrogen interaction sites were offset from the hydrogen nuclei as in the Williams prescription. ${ }^{29}$ As shown in Fig. 3(a), agreement between the experimental and the RISM structure functions is poor, with the latter damping too rapidly and out of phase at higher angle. While a change in temperature should improve the damping envelope, the phase shift implies short range packings of molecules in the RISM fluid that are more dense at $298 \mathrm{~K}$ than the actual packings in our cold clusters according to our diffraction patterns. As shown in Fig. 3(b), lowering the temperature to $148 \mathrm{~K}$ without changing the density of the RISM fluid gives a better agreement with the experimental intensity although the phase disparity at high angle is still evident. Resemblance between the RISM and experimental structure functions gets poorer at lower temperatures and higher densities. Accordingly, it is clear that RISM runs with temperature

TABLE III. Atom-atom nonbonded potential parameters" used in initial RISM calculations. Energy kJ/mol; distance $\AA$.

\begin{tabular}{|c|c|c|c|c|c|c|}
\hline \multicolumn{7}{|c|}{$\begin{array}{l}\text { Expression } \\
\qquad \begin{aligned} \phi(r) & =\mathrm{Ar}^{-6}+\mathrm{Be}^{-c r} \\
& =\epsilon \lambda(\lambda-6)^{-1}\left(-\left(r_{0} / r\right)^{6}+\left(6 e^{\lambda} / \lambda\right) e^{-\lambda r / r_{0}}\right\}\end{aligned}\end{array}$} \\
\hline Pair & $A$ & $10^{-4} B$ & $C$ & $r_{0}$ & $\epsilon$ & $\lambda$ \\
\hline $\mathrm{CC}$ & 1886 & 34.9 & 3.64 & 3.93 & 0.297 & 14.3 \\
\hline $\mathrm{CH}$ & 562 & 6.78 & 3.71 & 3.56 & 0.151 & 13.2 \\
\hline HH & 162 & 1.53 & 3.84 & 3.18 & 0.080 & 12.2 \\
\hline
\end{tabular}

"Close to but not identical to those of Williams, Ref. 29. Difference is $1 \%-$ $2 \%$ in $\lambda$. 


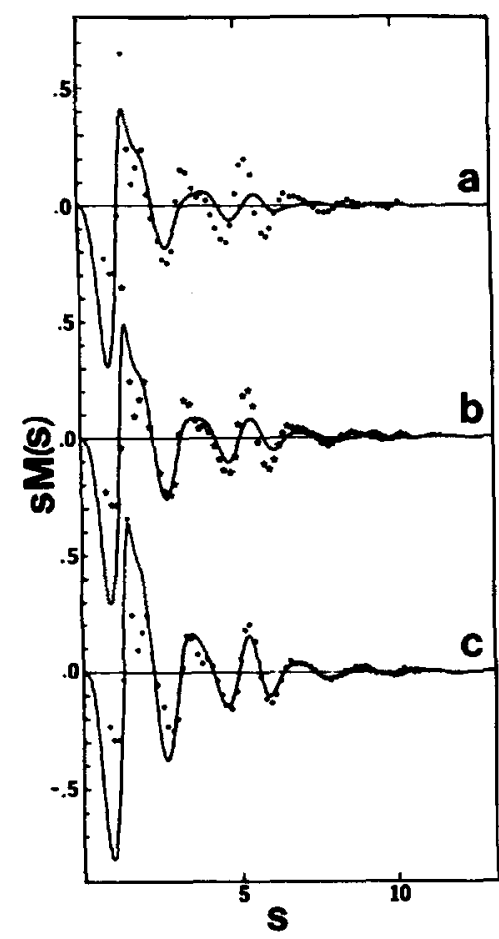

FIG. 3. Benzene intermolecular interference patterns. Points, electron diffraction experiment. Solid lines, calculated for electron diffraction by RISM liquid simulations. (a) Williams potentials (Ref. 29) $298 \mathrm{~K}$ temperature and density. (b) Williams potentials, $148 \mathrm{~K}$ temperature and $298 \mathrm{~K}$ density, a higher density worsening agreement with experiment. (c) Modified potentials with $6 \%$ larger atoms, $160 \mathrm{~K}$ and density $7.7 \%$ higher than value at $298 \mathrm{~K}$.

and density appropriate for our cold clusters are incapable of achieving close agreement with our diffraction intensities, if the Williams potential constants are retained.

To test whether modified intermolecular potentials can yield improved RISM results for benzene, we increased the parameter $r_{0}$ by $6 \%$ for each interacting pair while leaving the depth and the steepness of repulsion of the potentials as before. This increase in effective size of the atoms led to significantly better, though still imperfect, agreement with our experiments. The best agreement [Fig. 3(c)] was found at 160 $\mathrm{K}$, and $\rho=0.00725 / \AA^{3}$, a density about $12 \%$ lower than that derived for $160 \mathrm{~K}$ by extrapolation of values for liquid benzene. ${ }^{20}$ An optimization of atomic sizes was not investigated. Possibly a better compromise could be obtained with somewhat different atomic sizes but the characteristic discrepancy between experiment and RISM calculations shown in Fig. 3 would probably remain. The need to modify the Williams potentials is perhaps more an artifact of RISM than a deficiency of the Williams parameters.

\section{DISCUSSION \\ Inference of cluster temperature and size}

Diffraction patterns of amorphous phases contain far less information about structure, temperature, and particle size, unfortunately, than do patterns of crystallites. Nevertheless, the sharpness of height of the first interference feature is a measure of the length over which correlations in distance survive and, hence, is a measure of minimum particle size. This feature and the prominent feature at $s=5.3$ $\AA^{-1}$, which are both easily recognizable in all experimental and calculated diffraction patterns, are presumably dependent not only upon the size (diameter $D$ ), but also the temperature $(T)$, and fraction of vapor condensed $(f)$. For a given $T$ and $D$, the observed heights should be directly proportional to $f$. Certain regularities are apparent in a study of Fig. 4 of Ref. 1 and the values $h_{1}$ and $h_{5}$ in Table II of the present paper, where $h_{1}$ is the value of $s M(s)$ of the $1.3 \AA^{-1}$ diffraction ring and $h_{5}$ is taken as the trough to peak height, divided by 0.7 , of the $5.3 \AA^{-1}$ halo. The factor $1 / 0.7$ was arbitrarily introduced because $h_{5}$, at the characteristic temperatures of the experiments, then seemed to correlate approximately, with $f$ itself. At very much lower temperatures, calculated $h_{5}$ values for model clusters (bottom, Table II) rose markedly.

Consistency of $h_{5}$ values when temperature is in the broad range about $190 \mathrm{~K}$ provides weak evidence about $T$. An alternative predictive scheme to estimate the temperature of clusters is that proposed by Farges $e t$ al. ${ }^{30}$ who showed for condensation of neat monatomic and diatomic molecules into clusters that, in effect,

$$
T \approx 7.3 \Delta H_{v},
$$

where $\Delta H_{v}$ is the heat of vaporization at the boiling point in $\mathrm{kJ} / \mathrm{mol}$. This suggests a temperature of clusters from neat benzene of about $225 \mathrm{~K}$. Presumably, when a high concentration of carrier gas is available to remove heat, the temperature drops considerably below the Farges value. Nucleation calculation results listed in Table II suggest how much lower the temperature is when carrier gas is present. For the runs at intermediate mole fractions where clusters were condensed most efficiently, temperatures at maximum nucleation rate were in the range of $170-190 \mathrm{~K}$. Particles as small as those predicted by the simulations should efficiently exchange heat with the surrounding gaseous medium and leave the nozzle at $T_{\text {exit }}$ whose value, in the same runs, is $100-150$ $\mathbf{K}$, depending upon conditions. Appreciably lower temperatures result if less material is condensed and, hence, less heat has flowed into the gaseous medium. Values of $h_{5}$ weakly imply that temperatures are not as low as $100 \mathrm{~K}$. Similar hints were given in nucleation experiments with chalgogenide hexafluoride crystallites where lattice constants indicated that clusters tend to be significantly warmer than calculated nozzle exit temperatures. ${ }^{31}$ Possibly this is because turbulent mixing makes the clusters agglomerate into bigger snowballs which relax thermally more slowly than individual clusters would. Alternatively, it may be an effect of fractionation of the clusters toward, and the cooling carrier, away from the jet axis. A final estimate comes from the RISM simulations which gave closest agreement with experiment when $T \approx 160 \mathrm{~K}$. Not one of the above estimates of temperature is reliable but the estimates, each based on an entirely independent line of evidence, are broadly consistent with each other.

Calculated patterns for small clusters (Table II, bottom) give values of $h_{1}$ that very appreciably with size, as suggested in the opening paragraph of this section, and that are little influenced by temperature. Therefore, if the fraction condensed $f$ were known, $h_{1}$ should provide a measure of cluster size. If we gauge $f$ by $h_{5}$, the ratio $h_{1} / h_{5}$ should tend to normalize results to intensity per condensed material although we have now introduced a poorly defined function of temperature, $h_{5}$. A value of the ratio $h_{1} / h_{5}$ less than unity implies a very small cluster while a value approaching 2 suggests one of substantial size. It is seen in Table II that $h_{1} / h_{5}$ is correlated significantly but imperfectly with cluster size as calculat- 
ed in the nucleation simulations. At present no better values of size are available than the ones derived from nucleation calculations. Whether agglomeration into bigger clusters occurs may be revealed by light scattering measurements.

\section{Trends in nucleation and cluster growth}

Experimental indicators discussed above are in agreement with results of the nucleation simulations in the following trends, some of which have been seen in prior studies on other systems ${ }^{32}$ :

(1) At constant carrier stagnation pressure, reducing benzene mole fraction to small values causes $D$ and $f$ to decrease.

(2) At constant partial pressure of benzene, reducing carrier pressure causes $D$ and $f$ to decrease.

(3) At saturated vapor pressure of benzene $\left(20^{\circ} \mathrm{C}\right)$, a carrier pressure of 2-3 bar is needed for a nozzle similar to no. 6 to give reasonably complete condensation.

(4) As the molecular weight of carrier increases, $D$ and $f$ tend to increase.

These trends can be accounted for as follows. If the initial concentration of benzene is very low [case (1)], critical supersaturation is delayed until the cooling by expansion is considerable, at which point the gas is too dilute for clusters, once formed, to grow rapidly. If the carrier concentration is not large [case (2)], the heat capacity for removing heat of vaporization is small, and the medium stays too warm for efficient nucleation. Evaporation as well as condensation occurs for the warm clusters. Condition (3) is a satisfactory compromise between effects (1) and (2).

While the different carrier gases have appreciably different viscosities, Reynold's numbers in flow and, hence, different frictional dissipation rates, the dominant influence of carrier molecular weight $M$ is that of governing flow velocity. In frictionless flow, flow velocity at the throat of a Laval nozzle is sonic, ${ }^{18}$ with velocity $(\gamma R T / \bar{M})^{1 / 2}$. According to the present computer runs, the dependence on $\bar{M}$ is not far from that predicted for inviscid flow. Note that for inviscid flow as a function of distance $x$ along the nozzle, the profile $T(x)$ is well known to be independent of $\bar{M}$. Therefore, in first approximation, nucleation rate $J$ as a function of $x$ is independent of $M$. Clusters once formed, however, have more time to grow between $x$ and $x+\Delta x$, the higher is $M$ (the slower is the flow). Consequently, more heat is generated between $x$ and $x+\Delta x$, the higher is $M$. This shuts down the nucleation rate rapidly, so that fewer nuclei are formed for higher $M$. These nuclei have time to grow, deplete the vapor, and become large. For smaller $M, D$ is smaller partly because more nuclei are ultimately formed, and partly because the ones formed may have too little residence time to exhaust the vapor around them. Both effects are significant according to the simulations.

Another effect, not incorporated into the computer model, is that for condensate molecule as heavy as benzene, thermal accomodation coefficients between carrier and cluster increase considerably as $M$ increases from that of helium. ${ }^{33}$ Presumably, this effect would enhance the effects already mentioned. Acting in the same direction to produce cooler clusters with heavier carrier molecules is the fraction- ation noted in the Experimental section. Light carrier molecules separate from the clusters and contribute fewer cooling collisions. These effects on the cooling of clusters already formed may be of more importance in the production of low temperature phases observed elsewhere ${ }^{31}$ than in the present results.

\section{Aspects of the RISM results}

Results of the present calculations showed the same inadequacies of the RISM approach as noted by Narten, ${ }^{2}$ but in exaggerated degree. Our colder material exhibits a higher degree of ordering and our electron-optical sensitivity can discern it readily, both in the sharp features it imparts at small angles and the low amplitude features at higher angles. The RISM algorithm seems to be incapable of generating as rapidly modulated features in the intermolecular atom pair correlation functions as occur in the real packing of polyatomic molecules; RISM correctly establishes positions of the features but blurs them greatly. This can be shown by taking Fourier transforms of the observed and calculated electron diffraction structure functions $s M(s)$ as shown in Fig. 4. Here it should be pointed out that these Fourier transforms are not to be mistaken for the more common function $g(r)$ computed from X-ray or neutron intensities. This is because $s M(s)$ is strongly depressed at small $s$ by inelastic scattering, the scattering that dominates at small angles. Despite its other advantages, electron diffraction suffers from the disproportional contribution of inelastic scattering at very small angles that precludes the derivation of rigorous $g(r)$ functions with current technology. Electron diffraction analyses, then, have always relied most heavily upon comparisons between observed and calculated functions rather than upon the direct derivation of probability distributions. That this limitation is of little importance in assessing the RISM results can be seen in the transforms of Fig. 4. Experimental maxima at 3.9, 5.0, and $6.2 \AA$ agree well with those calculated from RISM electron diffraction structure functions $(4.0,4.9$, and $6.1 \AA)$. These peaks are governed mainly by $g_{c c}(r)$ which give maxima at 4.2, 5.0, and 6.1 $\check{\AA}$. Displacements of maxima arise from contributions by $g_{\mathrm{CH}}(r)$ and $g_{\mathrm{HH}}(r)$. Hydrogens contribute more to electron than to $\mathrm{x}$-ray intensities and their interatomic contacts are shorter than those of carbon.

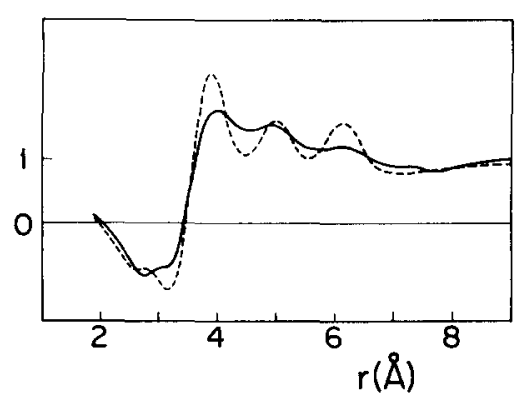

FIG. 4. Fourier sine transforms of intermolecular electron interference patterns for benzene. Solid line, calculated from RISM, Fig. 3(c) data. Dashed line, electron diffraction experiment blended over small angle range into RISM theory (to avoid termination ripples arising from inaccessible data, $s<0.8 \AA^{-1}$ ). Transforms are similar to but not equivalent to the intermolecular pair correlation function $g(r)$. 
Amplitudes of the RISM features can be seen to be grossly underestimated. It is doubtful that any reasonable pairwise additive potential energy function would appreciably change this type of misfit.

As far as structure is concerned, our clusters appear to be supercooled liquid with packing characteristics similar to those deduced in previous studies. Short range ordering, however, is more pronounced than predicted by RISM.

\section{CONCLUSIONS}

Four diverse, yet mutually important questions were addressed in this research. Can readily controlled clusters of polyatomic molecules suitable for diffraction studies be generated supersonically? Can their nucleation and growth be satisfactorily simulated by digital computation? Can the present approach be exploited to yield new information about liquid structure? Are available statistical treatments of liquid structure satisfactory? Preliminary answers applicable to the benzene system were found.

Homogeneous nucleation in nozzle flow can readily produce condensed matter for study and at reproducible conditions not easily achieved by other techniques. Large, freshly generated clusters of benzene discharged from the nozzle at various particle sizes and temperatures appear to correspond to supercooled bulk liquid. Computer simulations of the nucleation process and growth of the clusters based on classical nucleation theory successfully reproduce observed trends of benzene droplet size and fraction of vapor condensed as functions of pressure, concentration and molecular weight of carrier gas, and nozzle design. Even though this theory cannot be considered as quantitative, its predictions about cluster sizes and nucleation temperatures compare encouragingly with results of diffraction experiments in cases where clusters are crystalline and readily decipherable. ${ }^{31}$ In cases where clusters are amorphous, then, nucleation theory can provide a useful auxiliary means of estimating cluster properties.

Perhaps the greatest potential of the new technique for studying condensed phases lies in its ability to subject to examination the structure of liquids, and with a higher signal-to-noise ratio than is customarily attainable by other techniques. Few theoretical studies of liquid structure are available for comparison and none have been carried out on benzene using the most reliable methods (Monte Carlo, molecular dynamics) with potential functions of the quality invoked to reproduce crystallographic properties. ${ }^{29}$ Calculations adopting such functions were performed for liquid benzene in the present investigation, however, using the RISM algorithm of Chandler ${ }^{4}$ as modified by Johnson. ${ }^{7}$ These exhibited systematic deficiencies. First, in order to approximate experimental results, the RISM calculations required atomic sizes that were somewhat too large. Secondly, although distribution peaks in packing distances implied by RISM were well centered on the experimental peaks, they were far too diffuse. An indication of such discrepancies can be seen, to a lesser degree than in the present research on cold benzene, in other work. ${ }^{5-7,24,34}$ Further research to investigate the generality of the present findings would be profitable.

\section{ACKNOWLEDGMENTS}

This research was supported by a grant from the National Science Foundation. We thank A. H. Narten and E. Johnson for providing their version of the RISM routine. We gratefully acknowledge Harry Rinker for assistance with experiments and the University of Michigan Computing Center for a generous computer allocation.

${ }^{1}$ R. H. Heenan, E. J. Valente, and L. S. Bartell, J. Chem. Phys. 78, 243 (1983).

${ }^{2}$ A. H. Narten, J. Chem. Phys. 67, 2102 (1977).

${ }^{3}$ D. E. Williams, Acta Crystallogr. Sect. A 36, 715 (1980).

${ }^{4}$ L. J. Lowden and D. Chandler, J. Chem. Phys. 61, 5228 (1974).

${ }^{5}$ D. G. Montague, M. R. Chowdhung, J. C. Dore, and J. Reed, Mol. Phys. 50, 1 (1983); A. Habenschuss, E. Johnson, and A. H. Narten, J. Chem. Phys. 74, 5234 (1981).

${ }^{6}$ A. H. Narten, E. Johnson, and A. Habenschuss, J. Chem. Phys. 73, 1248 (1980).

${ }^{7}$ E. Johnson and R. P. Hazoume, J. Chem. Phys. 70, 1599 (1979).

${ }^{8}$ L. S. Bartell, R. H. Heenan, and M. Nagashima, J. Chem. Phys. 78, 236 (1983).

${ }^{9}$ See AIP document no. PAPS JCPSA-80-1451-13 for 13 pages of tables containing experimental intensities, background functions, and intermolecular scattering for plates discussed in this work. Order by PAPS number and journal reference from American Institute of Physics, Physics Auxiliary Publication Services, 335 East 45th Street, New York, N.Y. 10017 . The price is $\$ 1.50$ for each microfiche (98 pages) or $\$ 5.00$ for photocopies of up to 30 pages, and $\$ 0.15$ for each additional page over 30 pages. Airmail additional. Make checks payable to the American Institute of Physics.

${ }^{10}$ P. Pulay, R. Mawhorter, D. A. Kohl, and M. Fink, J. Chem. Phys. 79, 184 (1983); S. R. Goates and L. S. Bartell, ibid. 77, 1866, 1874 (1982).

"K. Tamagawa, T. Ijijima, and M. Kimura, J. Mol. Struct. 30, 243 (1976). ${ }^{12}$ B. J. C. Wu, NTIS report AD-775 257 (1974).

${ }^{13}$ P. M. Sherman, D. D. McBride, T. Chmielewski, T. H. Pierce, and E. Oktay, Aerospace Research Laboratories report ARL 69-0089 (1969); AIAA J. 8, 789 (1970).

${ }^{14}$ O. F. Hagena, Surf. Sci. 106, 101 (1981).

${ }^{15}$ B. J. C. Wu, P. P. Wegener, and G. D. Stein, J. Chem. Phys. 68, 308 (1978).

${ }^{16}$ K. Ostwatitsch, Z. Angew, Math. Mech. 23, 1 (1942).

${ }^{17}$ O. Abraham, J. H. Binn, B. G. DeBoer, and G. D. Stein, Phys. Fluids 24, $1017(1981)$.

${ }^{18}$ J. K. Vennard, Elementary Fluid Mechanics, 4th ed. (Wiley, New York, 1961).

${ }^{19}$ T. Stanton and J. Parnell, Philos. Trans. R. Soc. London, Ser. A 214, 199 (1914).

${ }^{20}$ C. L. Yaws, Physical Properties (McGraw-Hill, New York, 1977), pp. 214-222.

${ }^{21}$ O. R. Quale, Chem. Rev. 53, 439 (1953).

${ }^{22}$ H. L. Johnston and E. R. Grilly, J. Phys. Chem. 46, 948 (1942).

${ }^{23}$ G. Torchet, P. Schwartz, J. Farges, M. F. de Feraudy, and B. Raoult, J. Chem. Phys. (in press).

${ }^{24}$ A. H. Narten and A. Habenschuss, J. Chem. Phys. 75, 3073 (1981).

${ }^{25}$ G. E. Bacon, N. A. Curry and S. A. Wilson, Proc. R. Soc. London Ser. A 279, 98 (1964).

${ }^{26}$ A. H. Narten, J. Chem. Phys. 48, 1630 (1968).

${ }^{27}$ D. J. Evans and R. O. Watts, Mol. Phys. 32, 93 (1976).

${ }^{28}$ E. Johnson and R. P. Hazoume, J. Chem. Phys. 70, 1599 (1979).

${ }^{29}$ D. E. Williams, Acta Crystallogr. Sec. A 30, 71 (1974). To simplify calculations we deleted the electrostatic terms. Such long range interactions are not supposed to be important in RISM structure implications. See D. Chandler, Acc. Chem. Res. 7, 246 (1974).

${ }^{30} \mathrm{~J}$. Farges, M. F. de Feraudy, B. Raoult, and G. Torchet, Surf. Sci. 106, 95 (1981).

${ }^{31}$ E. J. Valente and L. S. Bartell, J. Chem. Phys. 79, 2683 (1983).

${ }^{32}$ O. Abaraham, S. Kim, and G. D. Stein, J. Chem. Phys. 75, 402 (1981).

${ }^{33}$ L. B. Adams, in Fundamentals of Gas-Surface Interactions, edited by $\mathbf{M}$. Saltsburg, J. N. Smith, and M. Rogers (Academic, New York, 1967) p. 346; D. Menzel and J. Kouptsidis, ibid., p. 493.

${ }^{34} \mathrm{D}$. Chandler, in The Liquid State of Matter: Fluids, Simple and Complex, edited by E. W. Montroll and J. L. Lebowitz (North Holland, Amsterdam, 1982). 